\title{
On spectral asymptotics of the Sturm-Liouville problem with self-conformal singular weight
}

\author{
U. R. Freiberg*, N. V. Rastegaev ${ }^{\dagger}$ \\ July 11, 2018
}

\section{Introduction}

We consider spectral asymptotics for the Neumann problem

$$
\left\{\begin{array}{l}
-y^{\prime \prime}=\lambda \mu y, \\
y^{\prime}(0)=y^{\prime}(1)=0,
\end{array}\right.
$$

where the weight $\mu$ is a self-conformal measure on a line.

Remark 1. It is well known, that the change of the boundary conditions causes rank two perturbation of the quadratic form corresponding to the problem. It follows from the general variational theory (see [1, §10.3]) that counting functions of the eigenvalues of boundary-value problems, related to the same equation, but different boundary conditions, cannot differ by more than 2 .

The problem of the eigenvalues asymptotic behavior for this problem goes back to the works of M. G. Krein (see, for example, [2]).

From [3] it follows that if the measure $\mu$ contains absolutely continuous component, its singular component does not influence the main term of the spectral asymptotic.

In the case of singular measure $\mu$ it follows from early works by M. G. Krein, that the counting function $N:(0,+\infty) \rightarrow \mathbb{N}$ of eigenvalues of the problem (1) admits the estimate $o\left(\lambda^{\frac{1}{2}}\right)$ instead of the usual asymptotics $N(\lambda) \sim C \lambda^{\frac{1}{2}}$ in the case of measure containing a regular component. (see, e.g., [4] or [5], and also [6] for similar results for higher ever order operators and better lower bounds for eigenvalues for some special classes of measures).

The problem is comparatively well-studied in the case of a self-similar (self-affine) measure. Exact power exponent in the case of self-similar measure was obtained in [7].

${ }^{*}$ Institut für Stochastik und Anwendungen, Universität Stuttgart, Pfaffenwaldring 57, D-70569 Stuttgart, Germany. E-mail: freiberg@mathematik.uni-stuttgart.de.

${ }^{\dagger}$ Chebyshev Laboratory, St. Petersburg State University, 14th Line 29b, 199178 Saint-Petersburg, Russia. E-mail: rastmusician@gmail.com. Supported by the joint SPbU and DFG grant No. 6.65.37.2017 
It is shown in [8] and [9] that the eigenvalues counting function of problem (11) for the self-similar weight has the asymptotics

$$
N(\lambda)=\lambda^{D} \cdot(s(\ln \lambda)+o(1)), \quad \lambda \rightarrow+\infty,
$$

where $D \in\left(0, \frac{1}{2}\right)$ and $s$ is a continuous $T$-periodic function, dependent on the choice of the weight $\mu$ (see also [10] for similar asymptotics in the case of an arbitrary even order differential operator, and [11] for similar results for problems containing two self-similar measures). A series of works [12, 13, 14] is dedicated to the fine properties of the function $s$ for incrementally generalized classes of self-similar measures.

The aim of this paper is to find the power exponent $D$ in the case of self-conformal measure with some special properties.

This paper has the following structure. Sect. 2 provides the necessary definitions of self-conformal measures, derives their properties and defines some restrictions. Sect. 3 introduces the formal boundary value problem and defines the spectrum under consideration. Sect. 4 gives the definition of the deformed self-similar measure establishes the spectral asymptotics for them and formulates the strong bounded distortion property, which is the main restriction on the self-conformal measures considered in this paper. Sect. 5 shows the connection between self-conformal measures with strong bounded distortion property and deformed self-similar measures, thus extending the spectral asymptotics to them.

We denote by $C$ different constants, the values of which are of no consequence.

\section{Self-conformal measures on a line}

Let $m \geq 2$. We say $/=\left(\varphi_{1}, \ldots, \varphi_{m} ; \rho_{1}, \ldots, \rho_{m}\right)$ is a conformal iterated function system on $[0,1]$, if:

1. $\varphi_{i}:[0,1] \rightarrow \varphi_{i}([0,1])$ is a $C^{1+\gamma}$ diffeomorphism for $\gamma>0$ and all $i=1, \ldots, m$.

2. $\varphi_{i}((0,1)) \subset(0,1)$ and $\varphi_{i}((0,1)) \cap \varphi_{j}((0,1))=\varnothing$ for all $i, j=1, \ldots, m, i \neq j$.

3. $0<\left|\varphi_{i}^{\prime}(x)\right|<1$ for all $i=1, \ldots, m$ and all $x \in[0,1]$.

4. Positive numbers $\rho_{i}$ are such, that $\sum_{i=1}^{m} \rho_{i}=1$.

Remark 2. Clearly, the conformity property is redundant on a line, but we use the same terminology as in multidimensional case for compatibility. For multidimensional definition on a smooth Riemannian manifold see [17]. For more general definitions in a complete metric space, see [16].

Without loss of generality we assume, that $\varphi_{i}$ are numbered in ascending order, i.e. $\varphi_{i}(x) \leq \varphi_{i+1}(y)$ for all $x, y \in[0,1], i=1, \ldots, m-1$. We define boolean values $e_{i}$ as follows:

$$
e_{i}= \begin{cases}0, & \varphi_{i}(0)<\varphi_{i}(1), \\ 1, & \varphi_{i}(0)>\varphi_{i}(1) .\end{cases}
$$


As such, $e_{i}=1$ when $\varphi_{i}$ changes the orientation of the segment.

We define the operator $\mathcal{S}$ on the space $L_{\infty}[0,1]$ as follows:

$$
\mathcal{S}(f)=\sum_{i=1}^{m}\left(\chi_{\varphi_{i}([0,1])}\left(e_{i}+(-1)^{e_{i}} f \circ \varphi_{i}^{-1}\right)+\chi_{\left\{x>\varphi_{i}\left(1-e_{i}\right)\right\}}\right) \rho_{i} .
$$

Lemma 1. $\mathcal{S}$ is a contraction mapping on $L_{\infty}[0,1]$.

Proof.

$$
\left\|\mathcal{S}\left(f_{1}\right)-\mathcal{S}\left(f_{2}\right)\right\|_{\infty}=\left\|\sum_{i=1}^{m}\left(\left(f_{1}-f_{2}\right) \circ \varphi_{i}^{-1}\right) \chi_{\varphi_{i}([0,1])} \rho_{i}\right\|_{\infty}=\max _{i} \rho_{i} \cdot\left\|f_{1}-f_{2}\right\|_{\infty} .
$$

We note, that $\max _{i} \rho_{i}<1$, which proves the lemma.

Hence, by the Banach fixed-point theorem there exists a (unique) function $C \in$ $L_{\infty}[0,1]$ such that $\mathcal{S}(C)=C$. Function $C(t)$ could be found as the uniform limit of the sequence $\mathcal{S}^{k}(f)$ for $f(t) \equiv t$, which allows us to assume that it is continuous and monotone, and also $C(0)=0, C(1)=1$. The derivative of the function $C(t)$ in the sense of distributions is a measure $\mu$ without atoms, invariant with respect to / in the sense of Hutchinson (see [16]), i.e. it satisfies the relation

$$
\mu(E)=\sum_{i=1}^{m} \rho_{i} \cdot \mu\left(\varphi_{i}^{-1}(E)\right)
$$

for any measurable set $E$.

Definition 1. We call $\mu$ self-conformal measure and denote it

$$
\mu:=\mu\left(\varphi_{1}, \ldots, \varphi_{m} ; \rho_{1}, \ldots, \rho_{m}\right) .
$$

Remark 3. For a fixed measure $\mu$ the choice of iterated function system is not unique. Also, the definition does not require the function system to be conformal. For example, we will define measures using $W_{\infty}^{1}$ diffeomorphisms later. However, we call measure $\mu$ self-conformal only when it is possible to choose appropriate $C^{1+\gamma}$ diffeomorpisms to define it.

Lemma 2. Let us define

$$
\Phi(E):=\bigcup_{i=1}^{m} \varphi_{i}(E)
$$

and let $\left|\Phi^{k}([0,1])\right| \rightarrow 0$ as $k \rightarrow \infty$. Then measure $\mu$ is singular with respect to Lebesgue measure.

Proof. It is obvious, that $\operatorname{supp} \mu \subset \Phi^{k}([0,1])$ for every $k$, thus $|\operatorname{supp} \mu|=0$.

Lemma 3. Let $\operatorname{Lip} \sum_{i=1}^{m}\left|\varphi_{i}-\varphi_{i}(0)\right|<1$. Then $\left|\Phi^{k}([0,1])\right| \rightarrow 0$ as $k \rightarrow \infty$. 
Proof. From Lip $\sum_{i=1}^{m}\left|\varphi_{i}-\varphi_{i}(0)\right|=\alpha<1$ it follows by the definition of $\Phi$, that

$$
|\Phi([0, a])|=\operatorname{Lip} \sum_{i=1}^{m}\left|\varphi_{i}(a)-\varphi_{i}(0)\right| \leq \alpha|[0, a]|,
$$

thus for every measurable set $E$

$$
|\Phi(E)| \leq \alpha|E|
$$

and thus $\left|\Phi^{k}([0,1])\right| \leq \alpha^{k} \rightarrow 0$ as $k \rightarrow \infty$.

Corollary 1. Denote $\alpha_{i}:=\operatorname{Lip} \varphi_{i}=\left\|\varphi_{i}^{\prime}\right\|_{\infty}$ and let $\sum_{i=1}^{m} \alpha_{i}<1$. Then measure $\mu$ is singular with respect to Lebesgue measure.

Hereafter we always assume, that

$$
\sum_{i=1}^{m} \alpha_{i}=\sum_{i=1}^{m}\left\|\varphi_{i}^{\prime}\right\|_{\infty}<1
$$

In particular, $[0,1] \backslash \Phi([0,1])$ contains at least one interval.

Remark 4. If all diffeomorphisms $\varphi_{i}$ are linear functions, we call $\mu$ self-similar measure. For self-similar measures Lemma 3 means, that if $\Phi([0,1]) \neq[0,1]$, then $\mu$ is singular with respect to Lebesgue measure. More general ways to construct self-similar functions on a line are described in [15].

\section{Sturm-Liouville problem with self-similar weight}

We consider the formal boundary value problem

$$
\left\{\begin{aligned}
-y^{\prime \prime} & =\lambda \mu y \\
y^{\prime}(0) & =y^{\prime}(1)=0 .
\end{aligned}\right.
$$

We call the function $y \in W_{2}^{1}[0,1]$ its generalized solution if it satisfies the integral equation

$$
\int_{0}^{1} y^{\prime} \eta^{\prime} d x=\lambda \int_{0}^{1} y \eta d \mu(x)
$$

for any $\eta \in W_{2}^{1}[0,1]$. Substituting functions $\eta \in \stackrel{\circ}{W}_{2}^{1}[0,1]$ into the integral equation, we establish that the derivative $y^{\prime}$ is a primitive of a singular measure without atoms $\lambda \mu y$, thus $y \in C^{1}[0,1]$.

We denote by $\lambda_{n}(\mu)$ the eigenvalues of the problem (44) numbered in ascending order, and by

$$
N(\lambda, \mu):=\#\left\{n: \lambda_{n}(\mu)<\lambda\right\}
$$

their counting function. 


\section{Deformed self-similar measures}

Consider $S_{i}:[0,1] \rightarrow I_{i}$ - a set of affine (linear) contractions of $[0,1]$ onto nonintersecting subsegments $I_{i}$ of $[0,1]$. Denote by

$$
\mu_{0}:=\mu_{0}\left(S_{1}, \ldots, S_{m} ; \rho_{1}, \ldots, \rho_{m}\right)
$$

the self-similar measure generated by them. Let $g:[0,1] \rightarrow[0,1]$ be a $W_{\infty}^{1}$ diffeomorphism.

Definition 2. We define a deformed self-similar measure $\mu$ as

$$
\mu(E):=\mu_{0}(g(E))
$$

for every measurable set $E$.

Lemma 4. Let $\mu=\mu_{0} \circ g$ be a deformed self-similar measure and let $g$ be $a C^{1+\gamma}$ diffeomorphism for some $\gamma>0$. Then $\mu$ is a self-conformal measure.

Proof. It is clear, that

$$
\mu(E)=\mu_{0}(g(E))=\sum_{i=1}^{m} \rho_{i} \cdot \mu_{0}\left(S_{i}^{-1}(g(E))\right)=\sum_{i=1}^{m} \rho_{i} \cdot \mu\left(\left(g^{-1} \circ S_{i} \circ g\right)^{-1}(E)\right),
$$

thus

$$
\mu=\mu\left(\varphi_{1}, \ldots, \varphi_{m} ; \rho_{1}, \ldots, \rho_{m}\right),
$$

where $\varphi_{i}:=g^{-1} \circ S_{i} \circ g$. It is clear, that $\varphi_{i}$ are $C^{1+\gamma}$ diffeomorphisms and the lemma is proved.

\section{Proposition 1. [7, Theorem 3.6]}

$$
N\left(\lambda, \mu_{0}\right) \asymp \lambda^{D},
$$

i.e. there exist constants $C_{1}, C_{2}>0$, such that for all $\lambda \geq 0$

$$
C_{1} \lambda^{D} \leq N\left(\lambda, \mu_{0}\right) \leq C_{2} \lambda^{D}
$$

where $D \in\left(0, \frac{1}{2}\right)$ is the only solution of

$$
\sum_{i=1}^{m}\left(\rho_{i}\left|I_{i}\right|\right)^{D}=1
$$

Theorem 1. Let $\mu$ be a deformed self-similar measure. Then

$$
N(\lambda, \mu) \asymp N\left(\lambda, \mu_{0}\right),
$$

i.e. there exist constants $C_{1}, C_{2}$, such that for all $\lambda \geq 0$

$$
C_{1} N\left(\lambda, \mu_{0}\right) \leq N(\lambda, \mu) \leq C_{2} N\left(\lambda, \mu_{0}\right) .
$$


Proof. Consider $y \in W_{2}^{1}[0,1]$ and consider $z=y \circ g \in W_{2}^{1}[0,1]$. Note, that

$$
\int_{0}^{1} y^{2}(x) d \mu_{0}(x)=\int_{0}^{1} y^{2}(g(x)) d \mu(x)=\int_{0}^{1} z^{2}(x) d \mu(x) .
$$

Note, also, that by changing the variable $t:=g(x)$ we obtain

$$
\int_{0}^{1}\left|z^{\prime}(x)\right|^{2} d x=\int_{0}^{1}\left|(y(g(x)))^{\prime}\right|^{2} d x=\int_{0}^{1}\left|y^{\prime}(g(x))\right|^{2}\left(g^{\prime}(x)\right)^{2} d x=\int_{0}^{1}\left|y^{\prime}(t)\right| g^{\prime}\left(g^{-1}(t)\right) d t .
$$

Since $g$ is $W_{\infty}^{1}[0,1]$ diffeomorphism, there exist constants $q, Q>0$, such that

$$
q<\left|g^{\prime}(x)\right|<Q
$$

Hence, from (6) it follows, that

$$
q \int_{0}^{1}\left|y^{\prime}(x)\right|^{2} d x \leq \int_{0}^{1}\left|z^{\prime}(x)\right|^{2} d x \leq Q \int_{0}^{1}\left|y^{\prime}(x)\right|^{2} d x
$$

thus, from (5) and (7), using Courant-Fischer-Weyl min-max principle, we obtain

$$
q \lambda_{n}\left(\mu_{0}\right) \leq \lambda_{n}(\mu) \leq Q \lambda_{n}\left(\mu_{0}\right)
$$

and

$$
N\left(q \lambda, \mu_{0}\right) \leq N(\lambda, \mu) \leq N\left(Q \lambda, \mu_{0}\right)
$$

Note, that by the Proposition 1

$$
N\left(q \lambda, \mu_{0}\right) \asymp N\left(\lambda, \mu_{0}\right), \quad N\left(Q \lambda, \mu_{0}\right) \asymp N\left(\lambda, \mu_{0}\right),
$$

and the theorem is proved.

Definition 3. Let's introduce the following notations:

$$
\Sigma_{k}=\{1, \ldots, m\}^{k}, \quad \Sigma_{*}=\bigcup_{i=0}^{\infty} \Sigma_{i}
$$

for a word $w=\left(i_{1}, i_{2}, \ldots, i_{k}\right) \in \Sigma_{k}$ we say $|w|=k$, denote

$$
\varphi_{w}=\varphi_{i_{1}} \circ \varphi_{i_{2}} \circ \ldots \circ \varphi_{i_{k}}
$$

and denote by $x_{w}$ the unique fixed point of $\varphi_{w}$ :

$$
\varphi_{w}\left(x_{w}\right)=x_{w}
$$

We will also use notation $\varphi^{[k]}$ for the composition of $k$ instances of function $\varphi$. 
Remark 5. The conformal iterated function system fulfils the bounded distortion property (see [17, Lemma 2.1]), i.e. there exists a constant $C \geq 1$, such that

$$
C^{-1} \leq\left|\frac{\varphi_{w}^{\prime}(x)}{\varphi_{w}^{\prime}(y)}\right| \leq C
$$

for all $x, y \in[0,1]$ and all $w \in \Sigma_{*}$.

For a deformed self-similar measure we have

$$
\varphi_{w}=g^{-1} \circ S_{w} \circ g,
$$

for every word $w \in \Sigma_{*}$, thus

$$
\varphi_{w}^{\prime}(x)=\frac{g^{\prime}(x)}{g^{\prime}\left(\varphi_{w}(x)\right)}\left|S_{w}([0,1])\right|
$$

for almost every $x \in[0,1]$. Note, that for $w=\left(i_{1}, i_{2}, \ldots, i_{k}\right) \in \Sigma_{k}$

$$
\left|S_{w}([0,1])\right|=\left|I_{i_{1}}\right| \cdot\left|I_{i_{2}}\right| \cdot \ldots \cdot\left|I_{i_{k}}\right|,
$$

does not depend on the order of elements of $w$, thus for $C=Q^{2} / q^{2}$ we have

$$
C^{-1} \leq\left|\frac{\varphi_{w}^{\prime}(x)}{\varphi_{\sigma w}^{\prime}(y)}\right| \leq C
$$

for all $x, y \in[0,1]$, all $w \in \Sigma_{*}$ and all permutations $\sigma \in \mathcal{S}_{|w|}$. We call this strong bounded distortion property. We are going to show in Sect. 5 , that this property is sufficient to prove, that self-conformal measure is a deformed self-similar measure.

Remark 6. It follows from (8), that if $g^{\prime}$ exists at the point $x_{i}$, then

$$
\left|\varphi_{i}^{\prime}\left(x_{i}\right)\right|=\left|I_{i}\right| .
$$

If $g^{\prime}\left(x_{i}\right)$ is not defined, then

$$
\left|I_{i}\right|=\lim _{k \rightarrow \infty} \sqrt[k]{\left|\varphi_{i}^{[k]}([0,1])\right|},
$$

so if $\varphi_{i}$ are $C^{1+\gamma}$ diffeomorphisms, then it is easy to see, that for all $x \in[0,1]$

$$
\left|\varphi_{i}^{\prime}\left(\varphi_{i}^{[k]}(x)\right)-\varphi_{i}^{\prime}\left(x_{i}\right)\right| \leq C \alpha_{i}^{k \gamma},
$$

thus

$$
\lim _{k \rightarrow \infty} \sqrt[k]{\mid \frac{\varphi_{i}^{[k]}([0,1])}{\left(\varphi_{i}^{\prime}\left(x_{i}\right)\right)^{k} \mid}}=1,
$$

and, yet again, we have

$$
\left|\varphi_{i}^{\prime}\left(x_{i}\right)\right|=\left|I_{i}\right| .
$$

Corollary 2. Let $\mu=\mu_{0} \circ g$ be a deformed self-similar measure and let $g$ be a $C^{1}$ diffeomorphism. Then

$$
N(\lambda, \mu) \asymp \lambda^{D},
$$

where $D \in\left(0, \frac{1}{2}\right)$ is the only solution of

$$
\sum_{i=1}^{m}\left(\rho_{i}\left|\varphi_{i}^{\prime}\left(x_{i}\right)\right|\right)^{D}=1
$$




\section{Deformation construction}

Consider a self-conformal measure

$$
\mu=\mu\left(\varphi_{1}, \ldots, \varphi_{m} ; \rho_{1}, \ldots, \rho_{m}\right)
$$

that satisfies the relation (3) and the strong bounded distortion property, and consider a self-similar measure

$$
\mu_{0}:=\mu_{0}\left(S_{1}, \ldots, S_{m} ; \rho_{1}, \ldots, \rho_{m}\right),
$$

that has the same structure as $\mu\left(S_{i}\right.$ changes orientation if and only if $\varphi_{i}$ changes orientation; $S_{i}([0,1])$ and $S_{i+1}([0,1])$ touch if and only if $\varphi_{i}([0,1])$ and $\varphi_{i+1}([0,1])$ touch $)$.

This section is concerned with two questions:

- If there exists a mapping $g$, such that $\mu=\mu_{0} \circ g$.

- If there exist $S_{1}, S_{2}, \ldots, S_{m}$, such that $g$ is a $W_{\infty}^{1}$-diffeomorphism.

\subsection{Construction}

For $i=1, \ldots, m-1$ denote by $U_{i}$ the intermediate interval (possibly empty) between $\varphi_{i}([0,1])$ and $\varphi_{i+1}([0,1])$, i.e.

$$
U_{i}=\left(c_{i}, d_{i}\right):=\left\{x \in[0,1]: \forall y \in[0,1] \varphi_{i}(y)<x<\varphi_{i+1}(y)\right\} .
$$

It is clear, that

$$
[0,1] \backslash \operatorname{supp} \mu=\bigcup_{i=1}^{m-1} \bigcup_{w \in \Sigma_{*}} \varphi_{w}\left(U_{i}\right) .
$$

Let's construct mapping $g$ explicitly by defining

$$
\forall w \in \Sigma_{*} \quad g\left(\varphi_{w}(0)\right)=S_{w}(0), \quad g\left(\varphi_{w}(1)\right)=S_{w}(1)
$$

connecting the dots linearly on each interval $\varphi_{w}\left(U_{i}\right)$, and thus everywhere outside supp $\mu$, and extending the definition continuously onto supp $\mu$.

By this definition,

$$
\mu=\mu_{0} \circ g
$$

and $g^{\prime}$ exists almost everywhere by Lebesgue measure.

Lemma 5. Denote

$$
\widetilde{\varphi}_{i}:=g^{-1} \circ S_{i} \circ g .
$$

Then $\widetilde{\varphi}_{i}$ is a $W_{\infty}^{1}$ diffeomorphism, $\widetilde{\varphi}_{i}=\varphi_{i}$ on $\operatorname{supp} \mu$,

$$
\forall w \in \Sigma_{*} \quad \widetilde{\varphi}_{w}(0)=\varphi_{w}(0), \quad \widetilde{\varphi}_{w}(1)=\varphi_{w}(1), \quad \widetilde{\varphi}_{w}\left(x_{w}\right)=x_{w}
$$

and

$$
\mu=\mu\left(\widetilde{\varphi}_{1}, \ldots, \widetilde{\varphi}_{m} ; \rho_{1}, \ldots, \rho_{m}\right)
$$


Proof. Function $g$ is continuous and strictly monotonous by definition, so $g^{-1}$ is also continuous, and so is $\widetilde{\varphi}_{i}$ for every $i$. Using (10) we obtain for all $w \in \Sigma_{*}$

$$
\widetilde{\varphi}_{w}(0)=g^{-1}\left(S_{w}(g(0))\right)=\varphi_{w}(0), \quad \widetilde{\varphi}_{w}(1)=g^{-1}\left(S_{w}(g(1))\right)=\varphi_{w}(1)
$$

thus $\widetilde{\varphi}_{i}=\varphi_{i}$ on $\operatorname{supp} \mu$, since both functions are continuous and every point of supp $\mu$ is a limit point of the set $\left\{\varphi_{w}(0), w \in \Sigma_{*}\right\}$. Fixed point $x_{w}$ is also a limit point of the set $\left\{\varphi_{w}(0), w \in \Sigma_{*}\right\}$, since $x_{w}=\lim _{k \rightarrow \infty} \varphi_{w}^{[k]}(0)$. Outside supp $\mu$ functions $\widetilde{\varphi}_{i}$ are linear on every interval, moreover, for all $i=1, \ldots, m, j=1, \ldots, m-1, v, w \in \Sigma_{*}$ we have

$$
\left|\widetilde{\varphi}_{v}^{\prime}\right| \equiv \frac{\left|\varphi_{v}\left(\varphi_{w}\left(U_{j}\right)\right)\right|}{\left|\varphi_{w}\left(U_{j}\right)\right|}=\frac{1}{\left|\varphi_{w}\left(U_{j}\right)\right|} \int_{\varphi_{w}\left(U_{j}\right)}\left|\varphi_{v}^{\prime}(t)\right| d t \quad \text { on } \varphi_{w}\left(U_{j}\right),
$$

which gives us the relation

$$
\left\|\widetilde{\varphi}_{i}^{\prime}\right\|_{\infty} \leq\left\|\varphi_{i}^{\prime}\right\|_{\infty}
$$

thus $\widetilde{\varphi}_{i} \in W_{\infty}^{1}$. Similarly, $\widetilde{\varphi}_{i}^{-1} \in W_{\infty}^{1}$, thus $\widetilde{\varphi}_{i}$ is a $W_{\infty}^{1}$ diffeomorphism.

\subsection{Smoothness}

We want to choose $S_{i}$ in such a way, that $g$ turns out to be $W_{\infty}^{1}$-diffeomorphism. Let's define

$$
S_{i}(x)=c_{i}+\varphi_{i}^{\prime}\left(x_{i}\right) x
$$

where $c_{i}$ are arbitrary, but chosen in such a way, that $\mu$ and $\mu_{0}$ have the same structure as described at the beginning of Sect. 5 .

Lemma 6. Let $\left(\varphi_{1}, \ldots, \varphi_{m}\right)$ satisfy the strong bounded distortion property. Then for every $w=\left(i_{1}, \ldots, i_{k}\right) \in \Sigma_{*}$ and every $x \in[0,1]$

$$
C^{-1} \leq\left|\frac{\widetilde{\varphi}_{w}^{\prime}(x)}{\varphi_{i_{1}}^{\prime}\left(x_{i_{1}}\right) \cdot \ldots \cdot \varphi_{i_{k}}^{\prime}\left(x_{i_{k}}\right)}\right| \leq C
$$

for some $C>0$.

Proof. From (11) we obtain, that since $\widetilde{\varphi}_{w}^{\prime}$ is an average of $\varphi_{w}^{\prime}$ on intervals outside $\operatorname{supp} \mu$, then for every $x \in[0,1]$ there exist some $x_{1}, x_{2} \in[0,1]$, such that

$$
\varphi_{w}^{\prime}\left(x_{1}\right) \leq \widetilde{\varphi}_{w}^{\prime}(x) \leq \varphi_{w}^{\prime}\left(x_{2}\right)
$$

Thus, it is sufficient to prove, that

$$
C^{-1} \leq\left|\frac{\varphi_{w}^{\prime}(x)}{\varphi_{i_{1}}^{\prime}\left(x_{i_{1}}\right) \cdot \ldots \cdot \varphi_{i_{k}}^{\prime}\left(x_{i_{k}}\right)}\right| \leq C
$$

for some $C>0$, every $w=\left(i_{1}, \ldots, i_{k}\right) \in \Sigma_{*}$ and every $x \in[0,1]$. Using strong bounded distortion property we conclude, that without loss of generality, we could assume, that $i_{1} \leq i_{2} \leq \ldots \leq i_{k}$. Then

$$
\varphi_{w}=\varphi_{1}^{\left[k_{1}\right]} \circ \ldots \circ \varphi_{m}^{\left[k_{m}\right]}
$$


for certain values of $k_{1}, \ldots, k_{m}$. By the estimate (9), for every $x \in[0,1]$ we have

$$
\prod_{j=0}^{k_{i}-1}\left(1-C \alpha_{i}^{j \gamma}\right) \leq\left|\frac{\left(\varphi_{i}^{\left[k_{i}\right]}\right)^{\prime}(x)}{\left(\varphi_{i}\left(x_{i}\right)\right)^{k_{i}}}\right| \leq \prod_{j=0}^{k_{i}-1}\left(1+C \alpha_{i}^{j \gamma}\right) .
$$

The products converge, since $\alpha_{i}<1, \gamma>0$. Now it is sufficient to note, that

$$
\left|\frac{\varphi_{w}^{\prime}(x)}{\varphi_{i_{1}}^{\prime}\left(x_{i_{1}}\right) \cdot \ldots \cdot \varphi_{i_{k}}^{\prime}\left(x_{i_{k}}\right)}\right|=\left|\frac{\left(\varphi_{1}^{\left[k_{1}\right]}\right)^{\prime}\left(\xi_{1}\right)}{\left(\varphi_{1}\left(x_{1}\right)\right)^{k_{1}}}\right| \cdot \ldots \cdot\left|\frac{\left(\varphi_{m}^{\left[k_{m}\right]}\right)^{\prime}\left(\xi_{m}\right)}{\left(\varphi_{m}\left(x_{m}\right)\right)^{k_{m}}}\right|,
$$

where $\xi_{i}:=\varphi_{i+1}^{\left[k_{i+1}\right]} \circ \ldots \circ \varphi_{m}^{\left[k_{m}\right]}(x)$, is a product of $m$ bounded terms.

Lemma 7. Let self-conformal measure

$$
\mu=\mu\left(\varphi_{1}, \ldots, \varphi_{m} ; \rho_{1}, \ldots, \rho_{m}\right)
$$

satisfy the relation (31) and the strong bounded distortion property. Then $\mu$ is a deformed self-similar measure, i.e. $\mu=\mu_{0} \circ g$, where

$$
\mu_{0}:=\mu_{0}\left(S_{1}, \ldots, S_{m} ; \rho_{1}, \ldots, \rho_{m}\right),
$$

$S_{i}$ are defined in (12) and $g$ is a $W_{\infty}^{1}$ diffeomorphism.

Proof. By definition

$$
\widetilde{\varphi}_{w}^{\prime}(x)=\frac{g^{\prime}(x)}{g^{\prime}\left(\widetilde{\varphi}_{w}(x)\right)}\left|S_{w}([0,1])\right|
$$

far a.e. $x \in[0,1]$ and all $w \in \Sigma_{*}$. From (12)

$$
\left|S_{w}([0,1])\right|=\left|\varphi_{i_{1}}^{\prime}\left(x_{i_{1}}\right)\right| \cdot \ldots \cdot\left|\varphi_{i_{k}}^{\prime}\left(x_{i_{k}}\right)\right|,
$$

thus

$$
\frac{g^{\prime}(x)}{g^{\prime}\left(\widetilde{\varphi}_{w}(x)\right)}=\frac{\widetilde{\varphi}_{w}^{\prime}(x)}{\varphi_{i_{1}}^{\prime}\left(x_{i_{1}}\right) \cdot \ldots \cdot \varphi_{i_{k}}^{\prime}\left(x_{i_{k}}\right)}
$$

and by Lemma 6 we have

$$
C^{-1} \leq\left|\frac{g^{\prime}(x)}{g^{\prime}\left(\widetilde{\varphi}_{w}(x)\right)}\right| \leq C
$$

Note, that for all $x, y \in U:=\bigcup_{i=1}^{m-1} U_{i}$

$$
C_{1}^{-1} \leq\left|\frac{g^{\prime}(x)}{g^{\prime}(y)}\right| \leq C_{1}
$$

for some $C_{1}>0$, since $g^{\prime}$ has only finite number of values inside $U$. Thus, since

$$
\left|\frac{g^{\prime}\left(\widetilde{\varphi}_{w_{1}}(x)\right)}{g^{\prime}\left(\widetilde{\varphi}_{w_{2}}(y)\right)}\right|=\left|\frac{g^{\prime}\left(\widetilde{\varphi}_{w_{1}}(x)\right)}{g^{\prime}(x)}\right| \cdot\left|\frac{g^{\prime}(y)}{g^{\prime}\left(\widetilde{\varphi}_{w_{2}}(y)\right)}\right| \cdot\left|\frac{g^{\prime}(x)}{g^{\prime}(y)}\right|,
$$

we conclude, that

$$
\left(C^{2} C_{1}\right)^{-1} \leq\left|\frac{g^{\prime}(x)}{g^{\prime}(y)}\right| \leq C^{2} C_{1}
$$

for all $x, y \in \bigcup_{i=1}^{m-1} \bigcup_{w \in \Sigma_{*}} \varphi_{w}\left(U_{i}\right)=[0,1] \backslash \operatorname{supp} \mu$, and $g$ is a $W_{\infty}^{1}$ diffeomorphism. 
Theorem 2. Let self-conformal measure

$$
\mu=\mu\left(\varphi_{1}, \ldots, \varphi_{m} ; \rho_{1}, \ldots, \rho_{m}\right)
$$

satisfy the relation (3) and the strong bounded distortion property. Then

$$
N(\lambda, \mu) \asymp \lambda^{D}
$$

where $D \in\left(0, \frac{1}{2}\right)$ is the only solution of

$$
\sum_{i=1}^{m}\left(\rho_{i}\left|\varphi_{i}^{\prime}\left(x_{i}\right)\right|\right)^{D}=1 .
$$

Every conformal iterated function system fulfils the bounded distortion property (see [17. Lemma 2.1]), but not every conformal iterated function system fulfils the strong bounded distortion property.

Example 1. Denote $w_{1}=(1,2,1,2)$ and $w_{2}=(2,1,1,2)$. Consider $C^{1+\gamma}$ diffeomorphisms $\varphi_{1}$ and $\varphi_{2}$, such that for some $\varepsilon>0$ we have

$$
\varphi_{w_{1}}^{\prime}(x)<(1-\varepsilon) \varphi_{w_{2}}^{\prime}(y)
$$

for every $x \in \varphi_{w_{1}}([0,1]), y \in \varphi_{w_{2}}([0,1])$. We could, for example, choose $\varphi_{1}(x)=a x$ for some $0<a<1$, and construct $\varphi_{2}(x)$ in such a way, that

$$
\begin{array}{ll}
\sqrt{1-\varepsilon} \cdot \varphi_{2}^{\prime}(y)>\varphi_{2}^{\prime}(x), & \forall x \in \varphi_{1}([0,1]), y \in \varphi_{2}([0,1]), \\
\sqrt{1-\varepsilon} \cdot \varphi_{2}^{\prime}(y)>\varphi_{2}^{\prime}(x), & \forall x \in \varphi_{1} \circ \varphi_{2}([0,1]), y \in \varphi_{1} \circ \varphi_{1} \circ \varphi_{2}([0,1]) .
\end{array}
$$

From this condition (15) follows, since

$$
\varphi_{w_{1}}^{\prime}(x)=a^{2} \varphi_{2}^{\prime}\left(\varphi_{1}\left(\varphi_{2}(x)\right)\right) \varphi_{2}^{\prime}(x)<a^{2}(1-\varepsilon) \varphi_{2}^{\prime}\left(\varphi_{1}\left(\varphi_{1}\left(\varphi_{2}(y)\right)\right)\right) \varphi_{2}^{\prime}(y)=(1-\varepsilon) \varphi_{w_{2}}^{\prime}(y)
$$

for every $x \in \varphi_{w_{1}}([0,1]), y \in \varphi_{w_{2}}([0,1])$. Note, that under this conditions

$$
\left|\frac{\left(\varphi_{w_{1}}^{[k]}\right)^{\prime}(x)}{\left(\varphi_{w_{2}}^{[k]}\right)^{\prime}(y)}\right|<(1-\varepsilon)^{k}
$$

for every $k \in \mathbb{N}, x \in \varphi_{w_{1}}([0,1]), y \in \varphi_{w_{2}}([0,1])$, which contradicts the strong bounded distortion property. 


\section{REFERENCES}

[1] Birman M. Sh., Solomyak M. Z. Spectral theory of self-adjoint operators in Hilbert space // Ed.2, Lan' publishers. - 2010. (in Russian); English translation of the 1st ed: Mathematics and its Applications (Soviet Series). D. Reidel Publishing Co., Dordrecht, 1987.

[2] Krein M. G. Determination of the density of the symmetric inhomogeneous string by spectrum// Dokl. Akad. Nauk SSSR - 1951. - V. 76, N. 3. - P. 345-348. (in Russian)

[3] Birman M. Sh., Solomyak M. Z. Asymptotic behavior of the spectrum of weakly polar integral operators// Mathematics of the USSR-Izvestiya. - 1970. - V. 4, N. 5. P. 1151-1168.

[4] Krein M. G., Kac I. S. A discreteness criterion for the spectrum of a singular string// Izvestiya Vuzov Matematika. - 1958. - N. 2. - P. 136-153. (in Russian)

[5] McKean H. P., Ray D. B. Spectral distribution of a differential operator// Duke Mathematical Journal. - 1962. - V. 29. - N. 2. - P. 281-292.

[6] Borzov V. V. On the quantitative characteristics of singular measures// Problems of math. physics. - 1970. - V. 4. - P. 42-47. (in Russian)

[7] Fujita T. A fractional dimention, self-similarity and a generalized diffusion operator // Taniguchi Symp. PMMP. Katata. - 1985. - P. 83-90.

[8] Solomyak M., Verbitsky E. On a spectral problem related to self-similar measures // Bull. London Math. Soc. - 1995. - V. 27, N 3. - P. 242-248.

[9] Kigami J., Lapidus M. L. Weyl's problem for the spectral distributions of Laplacians on p.c.f. self-similar fractals// Comm. Math. Phys. - 1991. - V. 158. - P. 93-125.

[10] Nazarov A. I. Logarithmic $L_{2}$-small ball asymptotics with respect to self-similar measure for some Gaussian processes// Journal of Mathematical Sciences (New York). - 2006. - V. 133, N. 3. - P. 1314-1327.

[11] Freiberg U. R. A Survey on Measure Geometric Laplacians on Cantor Like Sets // Arabian Journal for Science and Engineering. - 2003. - V. 28. - N. 1C. P. 189-198.

[12] Vladimirov A. A., Sheipak I. A. On the Neumann Problem for the Sturm-Liouville Equation with Cantor-Type Self-Similar Weight// Functional Analysis and Its Applications. - 2013. - V. 47, N. 4. - P. 261-270.

[13] Vladimirov A. A. Method of oscillation and spectral problem for four-order differential operator with self-similar weight// St. Petersburg Math. J. - 2016. - V. 27. N. 2. - P. 237-244. 
[14] Rastegaev N. V. On spectral asymptotics of the Neumann problem for the Sturm-Liouville equation with self-similar generalized Cantor type weight// J. Math. Sci. (N. Y.). - 2015. - V. 210. - N. 6. - P. 814-821.

[15] Sheipak I. A. On the construction and some properties of self-similar functions in the spaces $L_{p}[0,1] / /$ Mathematical Notes. - 2007. - V. 81, N. 6. - P. 827-839.

[16] Hutchinson J. E. Fractals and self similarity// Indiana Univ. Math. J. - 1981. V. 30, N 5. - P. 713-747.

[17] Patzschke N., Self-Conformal Multifractal Measures // Advances in Applied Mathematics. - 1997. - V. 19. - P. 486-513. 